\author{
International Journal of Mathematical Analysis \\ Vol. 9, 2015, no. 1, 15 - 18 \\ HIKARI Ltd, www.m-hikari.com \\ http://dx.doi.org/10.12988/ijma.2015.411363
}

\title{
On the Ideal Center of a Dual Vector Lattice
}

\author{
Serap Özcan and Ömer Gök \\ Y1ld1z Technical University, Faculty of Arts and Science \\ Department of Mathematics, Esenler, Davutpaşa, Istanbul, Turkey
}

Copyright (C) 2014 Serap Özcan and Ömer Gök. This is an open access article distributed under the Creative Commons Attribution License, which permits unrestricted use, distribution, and reproduction in any medium, provided the original work is properly cited.

\begin{abstract}
Let $X$ be a vector lattice with order dual $X^{\prime}$. In this paper, we investigate when $\operatorname{Orth}\left(X^{\prime}\right)$ is an ideal center in $X^{\prime}$. In [6], Toumi answers related question posed by Wickstead in [1]. This study is the dual version of the paper of Toumi in [6].
\end{abstract}

Mathematics Subject Classification: 46B42, 47B65

Keywords: Ideal Center, Vector Lattice, Orthomorphism

\section{Introduction}

Let $X$ be an Archimedean vector lattice. $\operatorname{Orth}(X)$ denotes the vector lattice of all orthomorphisms on $X . Z(X)$ denotes the sublattice of $\operatorname{Orth}(X)$ consisting of those $\pi$ for which there is non-negative real number $\lambda$ with $-\lambda x \leq \pi(x) \leq \lambda x$ for all $x \in X^{+}$(Positive cone of $X$ ). We say that $Z(X)$ is the ideal center of $X$. A vector lattice $X$ under an associative multiplication is said to be lattice-ordered algebra ( $\ell$-algebra) whenever the multiplication makes $X$ an algebra, in addition it satisfies the following property: If $x, y \in X^{+}$, then $x y \in X^{+}$,[2]. A lattice-ordered algebra $X$ is said to be an $f$-algebra when $x \wedge y=0$ implies $x z \wedge y=z x \wedge y=$ 0 for each $z \in X^{+}$. Note that any Archimedean $f$-algebra is commutative. Moreover, squares in an $f$-algebra are positive,[3].

If $X$ is an $f$-algebra, the mapping $\rho: X \rightarrow \operatorname{Orth}(X)$ defined by $\rho(f)=$ $\pi_{f}$ where $\pi_{f}(g)=f g$ for all $f, g \in X$ is an algebra and lattice homomorphism. If $\rho(f) \in Z(X), f$ is said to be bounded and if every element of $X$ is bounded, the $f$-algebra $X$ is said to be bounded,[5].

Let $X$ be a vector lattice and let $0 \leq v \in X$. If for every real number $\mathcal{E}>0$, there exists a natural number $n_{\mathcal{E}}$ such that $\left|x_{n}-x\right| \leq \mathcal{E} v$ for all $n \geq n_{\mathcal{E}}$, the sequ- 
ence $\left\{x_{n}\right\}_{n \geq 0}$ in $X$ is called $(v)$ relatively uniformly convergent to $x \in X$ which is denoted by $x_{n} \rightarrow x(v)$. That is, if $x_{n} \rightarrow x(v)$ for some $0 \leq v \in X$, then the sequence $\left\{x_{n}\right\}_{n \geq 0}$ is called (relatively) uniformly convergent to $x$. This is denoted by $x_{n} \rightarrow x(\mathrm{r} . \mathrm{u})$. A vector lattice is called relatively uniformly complete if every relatively uniform Cauchy sequence in $X$ has a unique limit. Relatively uniform limits are unique if $X$ is Archimedean. The relatively uniform topology is denoted by (r.u) topology. $X$ is called Dedekind complete, if every non-empty order bounded set has a supremum and an infimum in $X$. $X$ is called uniformly complete if $\sup \left\{\sum_{i=1}^{n} x_{i}: n \in N\right\}$ exists for every uniformly bounded sequence $\left(x_{n}\right)_{1}^{\infty} \subset$ $X^{+},[7]$.

Let $X$ be a topological algebra and $M(X)$ be the set of all maximal two sided ideals in $X$. The space $M(X)$ is equipped with the $h k$-topology: $S \subset M(X)$ is closed if $S=H(K(S))$, where $K(S)$ is the intersection of all ideals in $S$ and $H(I)=\{M \in M(X): I \subset M\}$ for any two-sided ideal $I$ of $X$. If $X$ is also equipped with a compatible topology we consider a subset $m(X)$ of $M(X)$ consisting of closed ideals. We use small letters $h$ and $k$ to indicate $H$ and $K$ restricted to $m(X)$.

A lattice norm $\|$.$\| on X$ is called an $M$-norm if $\|x \vee y\|=\max \{\|x\|,\|y\|\}$ for all $x, y \in X^{+}$.

Note that, for unexplained terminology and notation, we refer to the standart books [3] and [7]. Next theorem is seen in the work of Basly and Triki in [5] and [6].

Theorem 1 If $X$ is a relatively uniformly complete $f$-algebra, the following are equivalent:

i. $X$ is bounded.

ii. Every maximal modular ring ideal in $X$ is uniformly closed.

iii. Every maximal modular ring ideal in $X$ is the kernel of a lattice and algebra homomorphism $X \rightarrow R$.

iv. Every maximal modular ring ideal in $X$ is a maximal order ideal.

Theorem 2 [6] Consider a relatively uniformly complete unital $f$-algebra $X$. Then $M(X)=m(X)$, with respect to the relatively uniform topology, if and only if

i. Every proper finitely generated ring ideal is contained in a uniformly closed maximal ring ideal of $X$.

ii. $m(X)$ is compact in $h k$-topology.

Proposition 3 [6] Any maximal ring ideal of a relatively uniformly complete semiprime $f$-algebra $X$ is an order ideal.

Now, we will give the dual version of the study in [6].

Theorem 4 [6] Let $X$ be a vector lattice and $X^{\prime}$ be order dual of $X$. Then the following results are equivalent:

i. $\operatorname{Orth}\left(X^{\prime}\right)=Z\left(X^{\prime}\right)$. 
ii. $M\left(\operatorname{Orth}\left(X^{\prime}\right)\right)=m\left(\operatorname{Orth}\left(X^{\prime}\right)\right)$, with respect to the relatively uniform topology. iii. Every proper finitely generated ring ideal is contained in a uniformly closed maximal ring ideal of $\operatorname{Orth}\left(X^{\prime}\right)$ and $m\left(\operatorname{Orth}\left(X^{\prime}\right)\right)$ is compact in the $h k$-topology.

Proof: $\mathrm{i} \Rightarrow$ ii Because $X^{\prime}$ is Dedekind complete, $\operatorname{Orth}\left(X^{\prime}\right)$ is Dedekind complete. So, Orth $\left(X^{\prime}\right)$ is relatively uniformly complete. First, define the gauge function $P(\pi):=\inf \left\{\lambda \in R:-\lambda I_{X^{\prime}} \leq \pi \leq \lambda I_{X^{\prime}}\right\}$ for $\pi \in \operatorname{Orth}\left(X^{\prime}\right)$. Since $\operatorname{Orth}\left(X^{\prime}\right)=$ $Z\left(X^{\prime}\right), \quad P(\pi)$ is an $M$-norm. Moreover, $\left(\operatorname{Orth}\left(X^{\prime}\right), P\right)$ is a Banach lattice. $\operatorname{Orth}\left(X^{\prime}\right)$ is a unital $f$-algebra under composition, so $\operatorname{Orth}\left(X^{\prime}\right)$ is a Banach unital $f$-algebra. Because every maximal ring ideal in $\operatorname{Orth}\left(X^{\prime}\right)$ is uniformly closed, any maximal ring ideal in $\operatorname{Orth}\left(X^{\prime}\right)$ is closed, with respect to the norm topology.

ii $\Leftrightarrow$ iii From the Theorem 2, it is proven.

$\mathrm{ii} \Rightarrow \mathrm{i}$ Assume by way of contradiction that $Z\left(X^{\prime}\right) \subsetneq \operatorname{Orth}\left(X^{\prime}\right)$. There exists $\pi \in$ $\left(\operatorname{Orth}\left(X^{\prime}\right)\right)^{+}$such that $\pi \notin Z\left(X^{\prime}\right)$. Suppose that $\varphi=\pi \wedge I_{X^{\prime}} \in\left(\operatorname{Orth}\left(X^{\prime}\right)\right)^{+}$and $\omega_{n}=\pi-(\pi \wedge n \varphi)$, for all $n \in N$. Because of $\pi \notin Z\left(X^{\prime}\right)$, we get $\omega_{n}=\pi-$ $(\pi \wedge n \varphi)>0$. Then $\left\{\omega_{n}\right\}^{d}$ is proper uniformly closed order and ring ideal of $\operatorname{Orth}\left(X^{\prime}\right)$. Moreover, we have $\left\{\omega_{n}\right\}^{d} \subset\left\{\omega_{n+1}\right\}^{d}$, for all $n \geq 1$. Let $A=$ $\bigcup_{n \geq 1}\left\{\omega_{n}\right\}^{d}$, then $A$ is a proper ring ideal of $\operatorname{Orth}\left(X^{\prime}\right)$. Since $\left(I_{X^{\prime}}-\varphi\right) \wedge$ $(\pi-\varphi)=0$, we get $\psi\left(I_{X^{\prime}}-\varphi\right) \wedge(\pi-\varphi)=0$ for all $\psi \in \operatorname{Orth}\left(X^{\prime}\right)$. It follows that $\psi\left(I_{X^{\prime}}-\varphi\right)=\psi-\psi \varphi \in\left\{\omega_{1}\right\}^{d}$ and therefore, $\psi-\psi \varphi \in A$ for all $\psi \in$ $\operatorname{Orth}\left(X^{\prime}\right)$. Because there exists a closed maximal ring ideal $M$ such that $A \subset M$ and $M\left(\operatorname{Orth}\left(X^{\prime}\right)\right)=m\left(\operatorname{Orth}\left(X^{\prime}\right)\right), M$ is relatively uniformly closed order ideal of $\operatorname{Orth}\left(X^{\prime}\right)$. So, the vector lattice quotient $\operatorname{Orth}\left(X^{\prime}\right) / M$ is Archimedean.

Let $\pi_{n}=(\pi \vee n \varphi)-\pi$ for all $n \in N$. It follows that $\pi_{n} \omega_{n}=0$. Therefore, $\pi_{n} \in$ $\left\{\omega_{n}\right\}^{d} \subset A$. If we pass to the classes modulo $M$, we have $\overline{\pi_{n}}=\overline{(\pi \vee n \varphi)-\pi}=$ $(\bar{\pi} \vee \overline{n \varphi})-\bar{\pi}=\overline{0}$ for all $n \geq 1$ and so $\overline{0} \leq \overline{n \varphi} \leq \bar{\pi}$ for all $n \geq 1$. Since $\operatorname{Orth}\left(X^{\prime}\right) / M$ is Archimedean, it follows that $\varphi \in M$. Since the relation $\psi-\psi \varphi \in$ $A$, we get $\psi \in M$. Consequently we get the equation $M=\operatorname{Orth}\left(X^{\prime}\right)$ which is a contradiction. This completes the proof of the theorem.

Corollary 5 [6] If $X$ is Banach lattice then $\operatorname{Orth}\left(X^{\prime}\right)=Z\left(X^{\prime}\right)$.

Proof: Since $X$ is a Banach lattice, any orthomorphism $\pi$ on $X^{\prime}$ is continuous, [7]. On the other hand, since the modulus of $\pi$ exists [4], it shows that $\operatorname{Orth}\left(X^{\prime}\right)$ is a Banach unital $f$-algebra under composition. It is clear that every maximal ring ideal in $\operatorname{Orth}\left(X^{\prime}\right)$ is closed with respect to the norm topology,[4]. So, every maximal ring ideal in $\operatorname{Orth}\left(X^{\prime}\right)$ is uniformly closed. Then $M\left(\operatorname{Orth}\left(X^{\prime}\right)\right)=$ $m\left(\operatorname{Orth}\left(X^{\prime}\right)\right)$ with respect to the relatively uniform topology. From the equivalent conditions $\mathrm{ii} \Rightarrow \mathrm{i}$ in Theorem 4 , we get $\operatorname{Orth}\left(X^{\prime}\right)=Z\left(X^{\prime}\right)$.

Acknowledgment. This paper is supported by the research project 2014.01.03. DOP02. 


\section{References}

[1] A. W. Wickstead, Representation and duality of multiplication operators on Archimedean Riesz spaces, Compos. Math., 35(3), (1977), 225-238.

[2] C. B. Huijsmans and B. de Pagter, Ideal theory in f-algebras, Transactions of the American Matmematical Society, 269, no. 1, (1982), 225-245.

http://dx.doi.org/10.2307/1998601

[3] C. D. Aliprantis and O. Burkinshaw, Positive Operators, Springer, Dordrecht, (2006). http://dx.doi.org/10.1007/978-1-4020-5008-4

[4] H. G. Dales, A. T. M. Lau, D. Strauss, Banach algebras on semigroups and on their compactifications, Mem. Am. Math. Soc., 966(i-v), (2010), 165.

http://dx.doi.org/10.1090/s0065-9266-10-00595-8

[5] M. Basly, A. Triki, On uniformly closed ideals in f-algebras, In:2nd Conference, Function Spaces, Marcel Dekker, (1995), 29-33.

[6] M. A. Toumi, When orthomorphisms are in the ideal center, Positivity, 18, no.3, (2014), 579-583. http://dx.doi.org/10.1007/s11117-013-0263-3

[7] P. Meyer - Nieberg, Banach Lattices, Universitext, Springer, Berlin, Germany, (1991). http://dx.doi.org/10.1007/978-3-642-76724-1

Received: November 28, 2014; Published: January 6, 2015 